\title{
AN ALGORITHM FOR A MINIMUM COVER OF A GRAPH
}

ROBERT Z. NORMAN ${ }^{1}$ AND MICHAEL O. RABIN ${ }^{2}$

Introduction. This paper contains two similar theorems giving conditions for a minimum cover and a maximum matching of a graph. Both of these conditions depend on the concept of an alternating path, due to Petersen [2]. These results immediately lead to algorithms for a minimum cover and a maximum matching respectively.

The first part of the paper contains a noninductive proof of the minimum cover theorem (Theorem 1) and the resultant algorithm. Next we define a set of "level transformations" on the set of minimum covers; any minimum cover can be obtained from any other minimum cover by a finite sequence of level transformations (Theorem 2). Thus we have a procedure for finding all minimum covers: First, reduce any cover to a minimum cover by the algorithm; second, apply the level transformations to obtain all other minimum covers. The treatment of the maximum matching theorem and its corresponding algorithm, in the following section, closely parallels that given for minimum covers. The final section establishes a relationship between minimum covers and maximum matchings.

Both the minimum cover theorem and the maximum matching theorem were first proved by induction. The inductive proof of the maximum matching theorem, together with a discussion of its relevance to two similar theorems dealing with point-covers and pointmatchings, are given in Berge [1].

The problem of finding a simple algorithm for obtaining a minimum cover of a graph was proposed by Paul Roth as the one-dimensional instance of the more general question of finding a minimum cover for a cubical complex. This problem is a topological formulation of the synthesis of a switching system with minimum cost (cf. Roth [3]).

1. General definitions. Let $G$ be a graph. A set $C$ of edges of $G$ is a cover of $G$ if every vertex of $G$ is incident to an edge of $C$. We shall present a procedure for reducing a given cover to a minimum cover,

Presented to the Society October 26, 1957; received by the editors January 20, 1958.

${ }^{1}$ Supported in part by an Office of Naval Research Logistics Project at Princeton University.

2 Now at the Hebrew University in Jerusalem. 
using the concept of an alternating path first introduced by Petersen [2].

By a path of $G$ we mean a sequence of pairwise distinct successively adjacent edges of $G$. If $E$ is a set of edges of $G$, an alternating path of $(G, E)$ is a path whose edges are alternately in $E$ and not in $E$. The first and last edges of a path are its terminal edges. Its terminal vertices are the vertex incident to the first but not the second edge, and the vertex incident to the last but not the preceding edge.

Let $C$ be a cover of $G$. An alternating path of $(G, C)$ is a reducing path if

(1) its terminal edges are in $C$;

(2) its terminal vertices are incident to edges of $C$ which are not terminal edges of the path.

If $(G, C)$ possesses no reducing path, $C$ is called an irreducible cover of $G$. A cover with the fewest possible edges is a minimum cover.

2. Irreducible and minimum covers. It is easy to see that every minimum cover is irreducible. The converse of this statement is the main topic of this section.

Theorem 1. Every irreducible cover is a minimum cover.

This theorem gives rise to an algorithm for finding a minimum cover. For if $C$ is a nonminimum cover of $G$, it has a reducing path $\pi$. We can form a new cover $C^{\prime}$ from $C$ by replacing the edges of $\pi$ in $C$ with those edges of $\pi$ not in $C$. Then since $\pi$ begins and ends with edges of $C, C^{\prime}$ has one fewer edge than $C$. This process is repeated until an irreducible cover is formed; such a cover is a minimum cover.

To prove the theorem we shall make use of the following distance function. Let $E$ and $F$ be two sets of edges of a graph $G$. The distance $d(E, F)$ is defined as the number of edges of $E$ not in $F$.

Lemma 1. Let $C$ be an irreducible cover and let $C_{1}$ be a cover for which $d\left(C_{1}, C\right)=0$. Then $C_{1}=C$.

Proof. The assumption $d\left(C_{1}, C\right)=0$ asserts that every edge of $C_{1}$ is in $C$. Since $C_{1}$ is a cover, both vertices of any edge $e$ in $C-C_{1}$ would be covered by edges of $C_{1}$; thus $e$ is a reducing path of length 1 of $(G, C)$, contradicting the irreducibility of $C$.

In the following let $C$ be an irreducible cover of a graph $G$ and let $M$ be a minimum cover chosen so that $d(M, C)$ is a minimum. We contend that $M=C$.

Lemma 2. If $e$ is in $M-C$, then $e$ is not adjacent to any edge of $M$.

Proof. Let $e=\left\{v_{1}, v_{2}\right\}$ and assume that $v_{1}$ is incident to another 
edge $e_{1}$ of $M$. Let $e_{2}$ be an edge of $C$ incident to $v_{2}$. The set $M^{\prime}$ $=M \cup\left\{e_{2}\right\}-\{e\}$ is a cover. Since $e$ is in $M, M^{\prime}$ has no more edges than $M$, and hence it too is a minimum cover. Therefore $e_{2}$ is not in $M$ and $d\left(M^{\prime}, C\right)=d(M, C)-1$, contrary to the hypothesis that $d(M, C)$ is a minimum.

Proof of Theorem 1. Assume $d(M, C)>0$, so that $M-C$ is not empty. Consider the collection of all simple paths (i.e., those without repeated vertices) satisfying the conditions: (a) the edges of the path are alternately in $M-C$ and in $C$; (b) the terminal edges of the path are in $M-C$. The collection is nonempty since a single edge of $M-C$ is a path satisfying (a) and (b). Let $\pi=\left(e_{0}, \cdots, e_{n}\right)$, $e_{i}=\left\{v_{i}, v_{i+1}\right\}$, be a path of maximal length in the collection. The vertex $v_{0}$ is covered by an edge $e_{0}^{\prime}=\left\{u_{0}, v_{0}\right\}$ in $C$. Now $u_{0}=v_{n}$ is impossible because then $M^{\prime}=M \cup\left\{e_{0}^{\prime}, e_{1}, e_{3}, \cdots, e_{n-1}\right\}-\left\{e_{0}, e_{2}, \cdots, e_{n}\right\}$ would be a minimum cover with $d\left(M^{\prime}, C\right)<d(M, C)$. Similarly, there is an edge $e_{n}^{\prime}=\left\{u_{n}, v_{n}\right\}$ in $C$ such that $u_{n} \neq v_{0}$.

We contend that $u_{0}$ and $u_{n}$ are covered by edges of $C$ other than $e_{0}^{\prime}$ and $e_{n}^{\prime}$. If $u_{0}=v_{k}$ for some $k$, then $k<n$, and one of the edges $e_{k-1}$ or $e_{k}$ is in $C$. Otherwise $u_{0}$ is incident to no edge of $\pi$ and we proceed as follows: Let $e_{0}^{\prime \prime}$ be an edge of $M$ incident to $u_{0}$. By Lemma 2, $e_{0}^{\prime \prime} \neq e_{0}^{\prime}$. But now $e_{0}^{\prime \prime}$ must be in $C$, for otherwise the path $\left(e_{0}^{\prime \prime}, e_{0}^{\prime}, e_{0}, e_{1}, \cdots, e_{n}\right)$ is a path longer than $\pi$ satisfying (a) and (b). Hence again $u_{0}$ is covered by an edge of $C$ different from $e_{0}^{\prime}$ and $e_{n}^{\prime}$. A similar argument holds for $u_{n}$.

The path $\left(e_{0}^{\prime}, e_{0}, e_{1}, \cdots, e_{n}, e_{n}^{\prime}\right)$ is therefore a reducing path of $(G, C)$, contradicting the irreducibility of $C$. Thus $d(M, C)=0$, and by Lemma $1, M=C$.

3. Level transformations and minimum covers. Motivated by the previous proof, we are led to define two operations, $T_{1}$ and $T_{2}$, called level transformations which, when applied to a minimum cover, transform it into a new minimum cover. Application of a $T_{1}$-transformation to any cover $C$ consists of picking a path $\left(e_{1}, e_{2}, e_{3}\right)$ of three edges such that $e_{2}$ and $e_{3}$ are in $C$, and forming the cover $T_{1}(C)=C \cup\left\{e_{1}\right\}$ $-\left\{e_{2}\right\}$. Application of a $T_{2}$-transformation to a cover $C$ consists of picking a circuit $\left(e_{1}, e_{2}, \cdots, e_{2 n}\right)$ such that for all $k, e_{2 k-1}$ is in $C$ and $e_{2 k}$ is not in $C$, and forming the cover

$$
T_{2}(C)=C \cup\left\{e_{2}, e_{4}, \cdots, e_{2 n}\right\}-\left\{e_{1}, e_{3}, \cdots, e_{2 n-1}\right\} .
$$

A $T_{1}$-transformation may decrease the number of edges of a cover by one; a $T_{2}$-transformation always leaves the number of edges invariant. When applied to a minimum cover, $M$, however, both $T_{1}(M)$ and $T_{2}(M)$ have the same cardinality as $M$, and are thus both mini- 
mum covers. Since the most interesting applications of $T_{1}$ and $T_{2}$ are to minimum covers, the use of the term "level transformation" is justified.

Examining the proofs of Lemmata 1 and 2 and the proof of the main theorem, one immediately sees that they actually entail the following statement: Let $C$ be an irreducible cover and let $\Gamma$ be a collection of covers closed with respect to $T_{1}$ and $T_{2}$-transformations. If $M$ in $\Gamma$ minimizes $d\left(M^{\prime}, C\right)$ for $M^{\prime}$ in $\Gamma$, then $M=C$. From this remark we immediately derive two results.

THeOREM 2. If $M_{1}$ and $M_{2}$ are minimum covers, then $M_{2}$ can be obtained by applying a (finite) sequence of level transformations to $M_{1}$.

Proof. Let $\Gamma$ be the collection of all minimum covers obtainable from $M_{1}$ by a finite sequence of level transformations; $\Gamma$ is certainly closed with respect to level transformations. As $M_{2}$ is a minimum cover, it must be irreducible; hence $M_{2}=M$ for some $M$ in $\Gamma$.

Theorem 3. The collection $\mathrm{TT}$ of all minimum covers of a graph $G$ can be characterized as the nonempty collection of covers of $G$ closed with respect to $T_{1}$ - and $T_{2}$-transformations in which all elements have the same cardinality.

Proof. Let $\Gamma$ be such a collection. Let $M$ be any minimum cover. Then by the closure of $\Gamma, M$ is in $\Gamma$. Since all elements of $\Gamma$ have the same cardinality, this implies $\Gamma \subset \mathfrak{T}$. That $\Gamma=\mathfrak{M}$ now follows from Theorem 2.

4. Unaugmentable and maximum matchings. A set $P$ of edges of a graph $G$ is a matching if no two of its edges are adjacent. An augmenting path of $(G, P)$ is an alternating $(G, P)$ path whose terminal vertices are incident to no edges of $P$. Such a path is easily seen to be a simple path. If $(G, P)$ has no augmenting path, $P$ is called $u n$ augmentable. A matching with the greatest possible number of edges is maximum. Of course every maximum matching is unaugmentable. The converse of this theorem is a theorem due to Berge [1] which gives rise to an algorithm similar to the one just discussed.

TheOREM 4 (BERGE). Every unaugmentable matching is maximum.

Proof. Let $P$ be an unaugmentable matching and let $M$ be a maximum matching for which $d(P, M)$ is a minimum. Clearly, if $d(P, M)=0$ then $P=M$. We construct a path of maximum length whose edges are alternately in $P-M$ and $M$. Since $M$ is also unaugmentable, it cannot begin and end with edges of $P-M$. It has, there- 
fore, equally many edges of $P-M$ and $M$. Now from $M$ we can form a new maximum matching $M^{\prime}$ by exchanging the edges of the path in $M$ for those in $P$. Then $d\left(P, M^{\prime}\right)<d(P, M)$, contrary to the minimality of $d(P, M)$.

5. Relations between covers and matchings. Let $C$ be a cover of a graph. We shall say that $C$ is minimal if no proper subset of it is a cover. Similarly, a matching is maximal if no proper superset of it is a matching. Let $C$ be a minimal cover of $G$. For each vertex $v_{i}$ of $G$ we define its $C$-degree $a_{i}$ as the number of edges of $C$ incident to $v_{i}$. Let $A$ be the set of multiply covered vertices of $G$, i.e., those for which $a_{i}>1$. For each vertex $v_{i}$ of $A$, we delete from $C a_{i}-1$ edges incident to $v_{i}$. The resulting set $P$ is a matching, since the $P$-degree of each point is at most 1 . However, $P$ need not be a maximal matching.

Let $P$ be a maximal matching. Then for each vertex of $P$-degree 0 , add to $P$ any incident edge of the graph. The result is, of course, a cover, but it need not be minimal. However, if we begin with a minimum cover $C$, this process produces a maximum matching, while if we start with a maximum matching, the process yields a minimum cover. To demonstrate, let $C$ be a minimum cover of a graph $G$, and let $P$ result from $C$ by elimination of $a_{i}-1$ edges incident to each multiply covered vertex $v_{i}$. Let $c$ denote the number of edges of $C$, and let $a=\sum\left(a_{i}-1\right)$ be the number of edges deleted in forming $P$. Thus $a$ is the number of vertices of $P$-degree 0 . If $P$ is not maximum there is a matching $P^{\prime}$ with $c-a+1$ edges, and the number of vertices of $P^{\prime}$-degree 0 is $a-2$. Form a cover by adding one edge incident to each vertex of $P$-degree 0 . This cover has $c-1$ edges, contrary to the hypothesis that $C$ is a minimum cover. The dual assertion is proved similarly.

\section{REFERENCES}

1. C. Berge, Two theorems in graph theory, Proc. Nat. Acad. Sci. U.S.A. vol. 43 (1957) pp. 842-844.

2. J. Petersen, Die Theorie der regulären Graphen, Acta Math. vol. 51 (1891) pp. 193-220.

3. J. P. Roth, Algebraic topological methods for the synthesis of switching systems I, Trans. Amer. Math. Soc. vol. 88 (1958) pp. 301-326.

Dartmouth College,

Princeton University and

International Business Machines Corporation 